\title{
Roles of microRNA-34a targeting SIRT1 in mesenchymal stem cells
}

\author{
Fengyun Zhang ${ }^{1,2}$, Jinjin Cui ${ }^{1,2}$, Xiaojing Liü ${ }^{3}$, Bo Lv ${ }^{1,2}$, Xinxin Liu ${ }^{1,2}$, Zulong Xie ${ }^{1,2}$ and Bo $\mathrm{Yu}^{1,2^{*}}$
}

\begin{abstract}
Introduction: Mesenchymal stem cell (MSC)-based therapies have had positive outcomes both in animal models of cardiovascular diseases and in clinical patients. However, the number and function of MSCs decline during hypoxia and serum deprivation $(\mathrm{H} / \mathrm{SD}$ ), reducing their ability to contribute to endogenous injury repair. MicroRNA-34a (miR-34a) is originally identified as a TP53-targeted miRNA that modulates cell functions, including apoptosis, proliferation, and senescence via several signaling pathways, and hence is an appealing target for MSC-based therapy for myocardial infarction.

Methods: Bone marrow-derived MSCs were isolated from 60-80 g male donor rats. Expression levels of miR-34a were determined by qRT-PCR. The roles of miR-34a in regulating cell vitality, apoptosis and senescence were investigated using the cell counting kit (CCK-8) assay, flow cytometric analysis of Annexin V-FITC/PI staining and senescence-associated $\beta$-galactosidase ( $\mathrm{SA}-\beta$-gal) staining, respectively. The expression of silent information regulator 1 (SIRT1) and forkhead box class $\mathrm{O} 3 \mathrm{a}$ (FOXO3a) and of apoptosis- and senescence-associated proteins in MSCs were analyzed by western blotting.

Results: The results of the current study showed that miR-34a was significantly up-regulated under H/SD conditions in MSCs, while overexpression of miR-34a was significantly associated with increased apoptosis, impaired cell vitality and aggravated senescence. Moreover, we found that the mechanism underlying the proapoptotic function of miR-34a involves activation of the SIRT1/FOXO3a pathway, mitochondrial dysfunction and finally, activation of the intrinsic apoptosis pathway. Further study showed that miR-34a can also aggravate MSC senescence, an effect which was partly abolished by the reactive oxygen species (ROS) scavenger, $\mathrm{N}$-acetylcysteine (NAC).
\end{abstract}

Conclusions: Our study demonstrates for the first time that miR-34a plays pro-apoptotic and pro-senescence roles in MSCs by targeting SIRT1. Thus, inhibition of miR-34a might have important therapeutic implications in MSC-based therapy for myocardial infarction.

\section{Introduction}

Ischemic heart disease (IHD) is the leading cause of death worldwide, and the resulting heart failure aggravates a country's health burden, particularly in developed countries [1]. Existing therapies are typically only able to slow, rather than reverse or prevent, the progression of heart failure. Furthermore, side effects remain the key

\footnotetext{
* Correspondence: dryu_hmu@163.com

'Key Laboratories of Education Ministry for Myocardial Ischemia Mechanism, The Second Affiliated Hospital of Harbin Medical University, 148 Baojian Road, Harbin 150086, P.R. China

${ }^{2}$ Department of Cardiology, The Second Affiliated Hospital of Harbin Medical University, 148 Baojian Road, Harbin 150086, P.R. China

Full list of author information is available at the end of the article
}

issue among these effective therapeutics [2]. In the last few years, bone marrow-derived mesenchymal stem cells (MSCs) have been found to function as one of the most suitable candidate seed cells for repairing and regenerating cardiomyocytes as well as restoring heart function, and have been widely studied [3,4]. Transplantation of MSCs leads to improved neovascularization of ischemic myocardium and inhibition of myocardial fibrosis, in addition to an increase in the secretion of prosurvival growth factors, including vascular endothelial growth factor, insulin-like growth factor, and hepatocyte growth factor [4, 5]. Despite these advantages, the poor survival rate of MSCs within the first few days after engrafting in 
infarcted hearts leads to only marginal functional improvement $[6,7]$. The harsh microenvironment of the infarcted myocardium produces high levels of oxidative stress, which makes a great contribution to cellular senescence and causes a sharp decline in the proliferative capacity and regenerative potential of MSCs [8]. There is thus an urgent need to identify a strategy to protect the cells against the hostile microenvironment created by ischemia, hypoxia, the inflammatory response, and pro-apoptotic and pro-senescence factors in order to improve the efficacy of MSC transplantation therapy.

MicroRNAs (miRNAs) are endogenous $\sim 22$-nucleotide RNAs that have emerged as negative regulators of gene expression, acting by targeting mRNAs for cleavage or translational repression, which occurs primarily through base pairing to the $3^{\prime}$ untranslated regions (UTRs) of target mRNAs $[9,10]$. With rapid advances in understanding of the regulation and roles of these small, noncoding RNAs in cardiac pathology, the therapeutic potential of regulation of miRNAs in cardiac disease settings is considered high $[9,11]$. Among the known miRNAs, expression of miR-34a was found to be elevated in mouse hearts after myocardial infarction (MI) [12] and in cardiac tissue from patients with heart disease [13], while inhibition of the expression of miR-34a alleviated apoptosis and senescence in myocardial cells [14, 15] and other cell lines [16-18]. However, the precise role of miR-34a in MSCs has not been unraveled to date.

Silent information regulator 1 (SIRT1), one of the potential targets of miR-34a [19], is an NAD-dependent deacetylase that regulates apoptosis in response to oxidative and genotoxic stress and plays a critical role in regulating cell cycle, senescence, and metabolism [19-21]. Initially identified as a longevity gene, SIRT1 has recently been implicated as a novel modulator of myocyte homeostasis, playing a key role in cardiomyopathy through the deacetylation of forkhead box $\mathrm{O}$ transcription factor 3a (FOXO3a) [20], which was also acknowledged as the transcription factor most closely related to the antioxidative protective effects associated with longevity [22, 23]. Further study showed in endothelial progenitor cells (EPCs) that SIRT1 has a pivotally protective role in the regulation of EPC apoptosis induced by $\mathrm{H}_{2} \mathrm{O}_{2}$, and that SIRT1 exerted this protective effect by inhibiting FOXO3a via FOXO3a ubiquitination and subsequent degradation [24]. However, it is entirely unknown whether SIRT1 affects biological activities in MSCs; and if so, what role FOXO3a plays in this process.

In the current study, we tested the hypothesis that overexpression of miR-34a increases cellular susceptibility to hypoxia and serum deprivation (H/SD)-induced apoptosis and aggravates cell senescence, and investigated the underlying mechanisms. The results showed that miR-34a played a crucial role in a plethora of biological processes via regulation of SIRT1/FOXO3a and the reactive oxygen species (ROS) pathway in MSCs. Inhibition of miR-34a might therefore be a promising therapeutic strategy for enhancing the biological functions of MSCs, thus demonstrating great therapeutic potential in clinical transplantation.

\section{Materials and methods}

\section{Ethics statement}

Male Sprague-Dawley rats weighing 60-80 g were obtained from the Laboratory Animal Science Department of the Second Affiliated Hospital of Harbin Medical University, Heilongjiang, P.R. China. All experimental animal procedures were approved by the Local Ethical Committee on Animal Care and Use of Harbin Medical University.

\section{MSC culture}

MSCs were cultured using the whole bone marrow adherent method, as described previously [25]. Briefly, total bone marrow was harvested from the femora of rats and was plated into $25 \mathrm{~cm}^{2}$ culture flasks at a concentration of $10^{6} \mathrm{cells} / \mathrm{ml}$ in Iscove's modified Dulbecco's medium (IMDM; HyClone-Thermo Fisher Scientific, Waltham, MA, USA) supplemented with $10 \%$ fetal bovine serum (FBS; Gibco, Grand Island, NY, USA) and $1 \%$ penicillin/ streptomycin (Beyotime Institute of Biotechnology, Nantong, China), at $37{ }^{\circ} \mathrm{C}$ with $5 \% \mathrm{CO}_{2}$. After 3 days of incubation, the medium was changed and then replaced every 3 days thereafter. Approximately 7-9 days after seeding, the cells became $70-80 \%$ confluent. The adherent cells were released from the dishes using $0.25 \%$ trypsin (Beyotime Institute of Biotechnology, Beijing, China) and expanded at a 1:2 or 1:3 dilution. MSCs at passage 3-5 were used in all experiments. MSCs were characterized by flow cytometric analysis for the expression of the typical markers CD90, CD29, and CD44 (all from BD Biosciences, Franklin Lakes, NJ, USA), and the absence of the hematopoietic markers CD45 (eBioscience, San Diego, CA, USA) and CD34 (Santa Cruz Biotechnology, Inc., Dallas, TX, USA), as reported previously [25].

\section{Cell viability assay}

The viability of MSCs was determined using the cell counting kit-8 (CCK-8) assay (Beyotime Institute of Biotechnology, Beijing, China) in accordance with the manufacturer's protocols. Cells were seeded into a 96-well plate (3000 cells per well), and their growth was measured following addition of $10 \mu \mathrm{l} \mathrm{CCK}-8$ into the culture medium for 2 hours. The absorbance of each well was quantified at $450 \mathrm{~nm}$ (Tecan Infinite M200 microplate reader; LabX, Austria). All data were calculated from triplicate samples. 


\section{MSC H/SD treatment}

Apoptosis was induced by H/SD in vitro, which was designed to mimic the in vivo conditions of ischemia in the myocardium and was carried out as reported previously [26]. Briefly, MSCs were washed and cultured with serum-free IMDM and incubated in a $5 \% \mathrm{CO}_{2} / 95 \% \mathrm{~N}_{2}$ incubator (controlled atmosphere chamber; PLAS-Labs, Lansing, MI, USA) for 6 hours. MSCs incubated in a $5 \% \mathrm{CO}_{2} / 95 \% \mathrm{O}_{2}$ incubator were used as the normoxic control and cultured in complete medium.

\section{Measurement of apoptosis}

Apoptosis was determined by staining cells with Annexin V-fluorescein isothiocyanate (FITC) and counterstaining with propidium iodide (PI) using the Annexin V-FITC/PI apoptosis detection kit (BD PharMingen, San Diego, CA, USA). Briefly, $0.5 \times 10^{6}$ cells were washed twice with phosphate-buffered saline (PBS) and stained with $5 \mu \mathrm{l}$ Annexin V-FITC and $5 \mu \mathrm{l}$ PI in $1 \times$ binding buffer (BD PharMingen) for 15 minutes at room temperature in the dark. Analyses were performed using bivariate flow cytometry in a BD FACSCanto II equipped with BD FACSDiva software (Becton-Dickinson, San Jose, CA, USA).

\section{Target gene prediction}

To identify the potential targets of miR-34a that mediated its pro-apoptotic role in MSCs, bioinformatics algorithms including miRBase (University of Manchester, Manchester, UK), TargetScan (David Bartel Lab, Whitehead Institute for Biomedical Research, MA, USA), PicTar (Rajewsky lab, NY, USA and Max Delbruck Centrum, Berlin, DE), and miRanda (Computational Biology Center at MSKCC, NY, USA) were applied.

\section{Cell transfection}

Before transfection, MSCs were replanted into six-well plates at a density of $2 \times 10^{5}$ cells per well and incubated overnight. For overexpression or inhibition of miR-34a, cells were transfected with different concentrations of miR-34a mimic or miR-34a inhibitor (both from Invitrogen, Carlsbad, CA, USA). For small interfering RNA (siRNA)-mediated gene knockdown, 100 nM SIRT1 siRNA (GenePharma Co., Ltd, Shanghai, China) was transfected into cells. As controls, cells were transfected with negative control (NC) mimic, NC inhibitor of miR-34a (both from Invitrogen,Carlsbad, CA, USA), or scrambled siRNA (siRNA-NT) of SIRT1 (GenePharma Co., Ltd, Shanghai, China). All miRNAs and siRNA were transfected into MSCs using a commercial transfection reagent (X-treme siRNA Transfection Reagent; Roche Applied Science, Penzberg, Germany) according to the manufacturer's protocol. Forty-eight or 72 hours after transfection, cells were harvested for further analysis.

\section{RNA extraction and quantitative RT-PCR}

For analysis of miR-34a expression, total RNA was extracted from the MSCs using TRizol reagent (Invitrogen, Shanghai, China) and reverse-transcribed into cDNA according to the manufacturer's instructions. Quantitative RT-PCR (qRTPCR) was performed to analyze the level of miR-34a with the miRcute miRNA First-Strand cDNA Synthesis Kit and the miRcute miRNA qPCR Detection Kit (SYBR Green; Tiangen, Beijing, China). All primers for miR-34a and U6 for the TaqMan miRNA assays were purchased from Gene Pharma. Relative gene expression levels were calculated by comparing the $\Delta \mathrm{Ct}$ values between control and experimental conditions for each PCR target using the following equation:

Relative gene expression $=2^{-(\Delta \mathrm{Ct} \text { sample- } \Delta \mathrm{CT} \text { control })}$.

For several other genes, total cellular RNA was isolated and reverse-transcribed using the transcriptor First-Stand cDNA Synthesis Kit, according to the manufacturer's instructions. qRT-PCR was carried out using the fast-start universal SYBR master and fluorescence quantitative PCR system [27]. The relative expression level of mRNAs was normalized to that of the internal control glyceraldehyde 3-phosphate dehydrogenase (GAPDH) using the $2^{-\Delta \Delta C t}$ cycle threshold method. Table 1 presents all related gene sequences.

\section{Measurement of mitochondrial membrane potential}

Mitochondrial membrane potential $(\Delta \Psi \mathrm{m})$ was measured using the JC-1 mitochondrial membrane potential assay kit (Beyotime Institute of Biotechnology, Beijing, China). JC-1 was widely used to assess changes in $\Delta \Psi \mathrm{m}$ and mitochondrial permeability transition. After designated treatment, cells were incubated with JC-1 working dye for 20 minutes, then washed twice with cold JC-1 staining buffer and visualized under a fluorescence microscope (DMI4000B; Leica, Wetzlar, Germany).

\section{ROS staining}

Cells were left untreated or pretreated with NAC, miR-34a mimic, siRNA-SIRT1, and miR-34a inhibitor separately or in combination and then stimulated with the diluted fluoroprobe $2^{\prime}, 7^{\prime}$-dichlorodihydrofluorescein diacetate (DCFHDA; Beyotime Institute of Biotechnology, Beijing, China) for 20 minutes at $37{ }^{\circ} \mathrm{C}$ with slight shaking every 5 minutes. After washing with serum-free culture medium, the cells were collected and examined by flow cytometry.

\section{Senescence-associated $\beta$-galactosidase staining}

MSC senescence was determined by in situ staining for senescence-associated $\beta$-galactosidase (SA- $\beta$-gal) using a senescence cell histochemical staining kit (Beyotime Institute of Biotechnology, Beijing, China). Briefly, MSCs after treatment were first fixed for 30 minutes at room temperature in fixation buffer. After washing with 
Table 1 Primers for quantitative RT-PCR and oligonucleotides

\begin{tabular}{|c|c|c|}
\hline Name & & Sequence \\
\hline \multicolumn{3}{|l|}{ Quantitative RT-PCR } \\
\hline \multirow[t]{2}{*}{ miR-34a } & Forward & 5'-AAGGCCACGGATAGGTCCATA-3' \\
\hline & Reverse & 5'-CGCTTTGGTGGTTCTGAAAGG-3' \\
\hline \multirow[t]{2}{*}{ SIRT1 } & Forward & 5'-AAGGCCACGGATAGGTCCATA-3' \\
\hline & Reverse & 5'-CGCTTTGGTGGTTCTGAAAGG-3' \\
\hline \multirow[t]{2}{*}{$\mathrm{FOXO3a}$} & Forward & 5'-TGCCGATGGGTTGGATTT-3' \\
\hline & Reverse & 5'-CCAGTGAAGTTCCCCACGTT-3' \\
\hline \multirow[t]{2}{*}{ U6 } & Forward & 5'-AAGGCCACGGATAGGTCCATA-3' \\
\hline & Reverse & 5'-CGCTTTGGTGGTTCTGAAAGG-3' \\
\hline \multirow[t]{2}{*}{$\beta$-actin } & Forward & 5'-CCCAGCACAATGAAGATCAAGATCAT-3' \\
\hline & Reverse & 5'-ATCTGCTGGAAGGTGTACAGCGA-3' \\
\hline \multicolumn{3}{|l|}{ Oligonucleotide } \\
\hline \multicolumn{2}{|l|}{ miR-34a mimic } & UGGCAGUGUCUUAGCUGGUUGUU CAACCAGCUAAGACACUGCCAUU \\
\hline \multicolumn{2}{|l|}{ NC mimic } & UUCUCCGAACGUGUCACGUTT ACGUGACACGUUCGGAGAATT \\
\hline \multicolumn{2}{|l|}{ miR-34a inhibitor } & UGGCAGUGUCUUAGCUGGUUGUU \\
\hline \multicolumn{2}{|l|}{ NC inhibitor } & CAGUACUUUUGUGUAGUACAA \\
\hline \multicolumn{2}{|l|}{ siRNA-SIRT1 } & GCACCGAUCCUCGAACAAUTT AUUGUUCGAGGAUCGGUGCTT \\
\hline \multicolumn{2}{|l|}{ siRNA-NT } & UUCUCCGAACGUGUCACGUTT ACGUGACACGUUCGGAGAATT \\
\hline
\end{tabular}

FOXO3a forkhead box O transcription factor 3a, miRNA microRNA, NC negative control, siRNA small interfering RNA, siRNA-NT scrambled siRNA, SIRT1 silent information regulator 1

PBS, cells were incubated with $\beta$-galactosidase staining solution for 16 hours at $37{ }^{\circ} \mathrm{C}$ without $\mathrm{CO}_{2}$. The reaction was stopped by the addition of PBS. Statistical analysis was performed by counting 600 cells for each sample.

\section{Protein extraction and western blot analysis}

After designated treatment, cells were washed twice with ice-cold PBS, and the total protein concentration was analyzed using the bicinchoninic acid assay (BCA; Beyotime Institute of Biotechnology, Beijing, China) according to the manufacturer's instructions. Total cell extracts $(50 \mu \mathrm{g}$ total protein) were resolved by sodium dodecyl sulfate (SDS)$10 \%$ polyacrylamide gel electrophoresis and transferred onto polyvinylidene difluoride (PVDF) membranes. Nonspecific binding was inhibited by incubating the membranes with $8 \%$ skimmed milk in Tris-buffered saline (TBS) with $0.5 \%$ Tween-20. Subsequently, membranes were incubated with antibodies against SIRT1, FOXO3a, cleaved-caspase 3 (Cl.CASP3), cleaved-polyADP-ribose polymerase 1 (Cl.PARP1), cytochrome $c$, P53 (all from Cell Signaling Technology, Danvers, MA, USA), p16, $\gamma$-H2A.X (both from Abcam, Cambridge, MA, USA), p21 (Santa Cruz, CA, USA), and $\beta$-actin (Zhongshan Golden Bridge Biotechnology, Beijing, China) overnight at $4{ }^{\circ} \mathrm{C}$ at an appropriate dilution (1:1000). The membranes were washed with TBS with Tween-20 (TBS-T) and then incubated with peroxidase-conjugated Affinipure goat anti-rabbit IgG $(\mathrm{H}+\mathrm{L})$ and anti-mouse $\operatorname{IgG}(\mathrm{H}+\mathrm{L})$-labeled secondary antibodies (Zhongshan Golden Bridge Biotechnology, Beijing, China) diluted at 1:5000 for 1 hour at $37^{\circ} \mathrm{C}$. Specific complexes were visualized on an X-ray film using ElectroChemi-Luminescence (ECL) detection with BeyoECL Plus (Beyotime Institute of Biotechnology, Beijing, China) following the manufacturer's protocol. All data were obtained in triplicate, independent experiments.

\section{Statistical analysis}

All data were analyzed using SPSS 19.0 (SPSS Inc., Chicago, IL, USA) and were expressed as mean \pm standard deviation (SD). Comparisons between two groups were performed using Student's $t$ test, while the significance of differences between three or more experimental groups was determined by one-way analysis of variance. $P<0.05$ was considered statistically significant.

\section{Results}

miR-34a expression increases under H/SD, and correlates with decreased cell survival and increased apoptosis qRT-PCR results showed that miR-34a was expressed in normal MSCs and that expression increased significantly when exposed to atmospheric conditions representing H/SD for 6 hours (Fig. 1a). We then used CCK-8 to evaluate the role of miR-34a in MSC survival, and found that overexpression of miR-34a reduced cell survival, while inhibition of miR-34a expression showed the opposite effect (Fig. 1b, c). Apoptosis has been identified as 


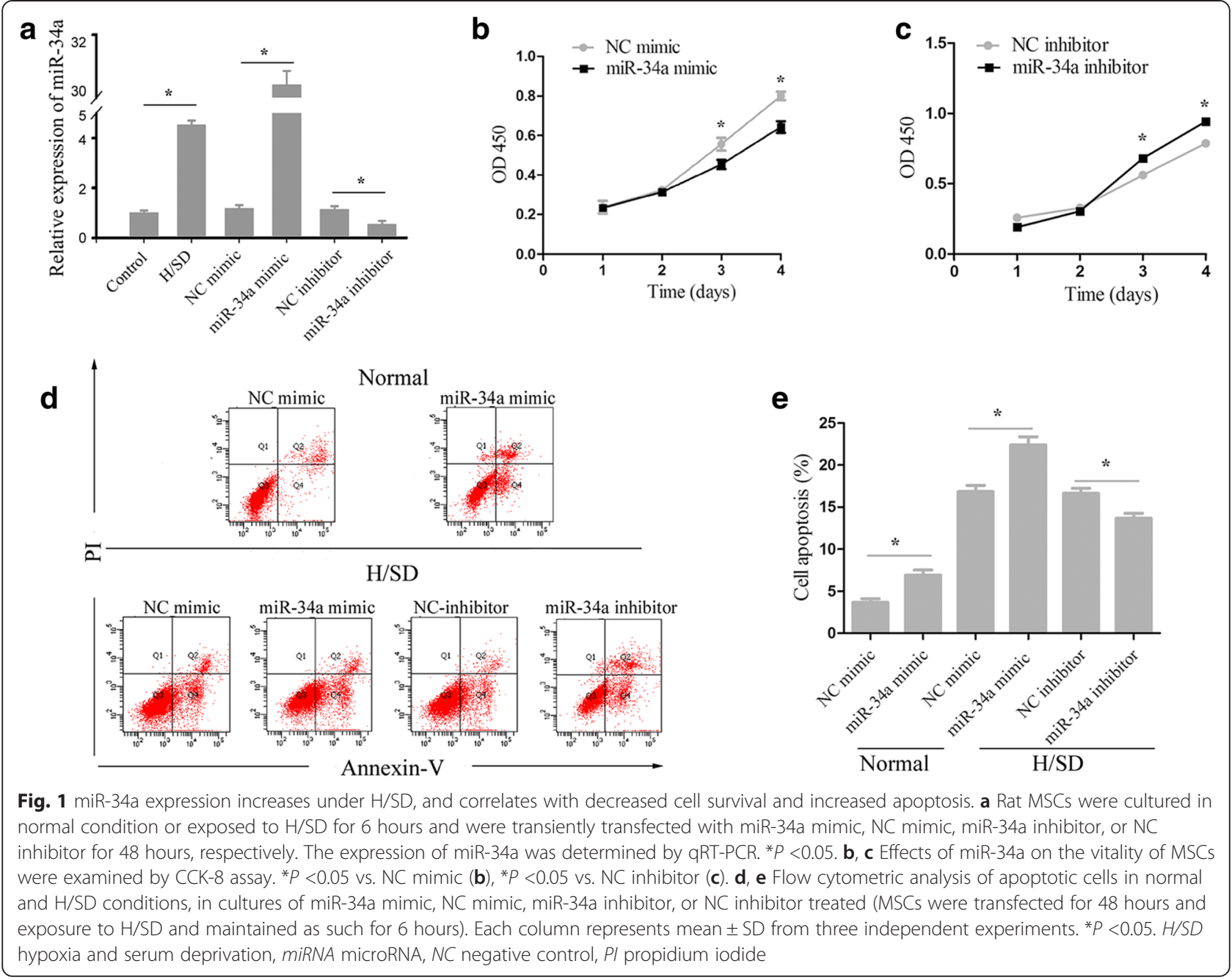

a major mechanism that reduces the survival rate after transplantation of MSCs into the harsh microenvironment of infarcted myocardium [26, 28]. To further determine the role of miR-34a in MSCs under conditions of $\mathrm{H} / \mathrm{SD}$, Annexin V-FITC/PI staining was performed. The results showed that miR-34a mimic-treated MSCs were significantly more apoptotic than the NC mimic group both in normal and H/SD conditions (Fig. 1d, e). However, when miR-34a was inhibited, MSCs showed better resistance to $\mathrm{H} / \mathrm{SD}$ than the $\mathrm{NC}$ inhibitor group (Fig. 1d, e). This supports our hypothesis that the decreased cell survival and increased apoptosis in MSCs are associated with overexpression of miR-34a.

\section{SIRT1 is a direct target of posttranscriptional repression by miR-34a}

Bioinformatics results suggested that SIRT1, identified as an apoptosis-associated gene, was the potential target of miR-34a. In addition, miRBase showed that the binding sites of miR-34a are evolutionarily conserved in both human and mouse (Fig. 2a). To test the hypothesis that
miR-34a regulates SIRT1 expression in MSCs from rats, we transfected MSCs with a miR-34a mimic or miR-34a inhibitor. As a control, some cells were transfected with $\mathrm{NC}$ mimic or NC inhibitor. Western blot analysis demonstrated a dose-dependent decrease in SIRT1 protein expression in miR-34a mimic-transfected cells compared with the NC mimic group (Fig. 2c). Notably, the inhibition of miR-34a in MSCs was concurrent with the increased expression of SIRT1 (Fig. 2d). However, qRT-PCR showed little difference in the expression levels of SIRT1 mRNA among the treatment groups. These data suggest that SIRT1 is likely to be targeted by miR-34a posttranscriptionally.

\section{miR-34a induces apoptosis by modifying SIRT1 and FOX03a expression}

After identifying SIRT1 as a direct target of miR-34a, we investigated whether knockdown of SIRT1 by siRNA (siRNA-SIRT1) induces apoptosis in MSCs. Similar to miR-34a mimic treatment, suppression of SIRT1 expression promoted apoptosis, revealed by flow cytometric 


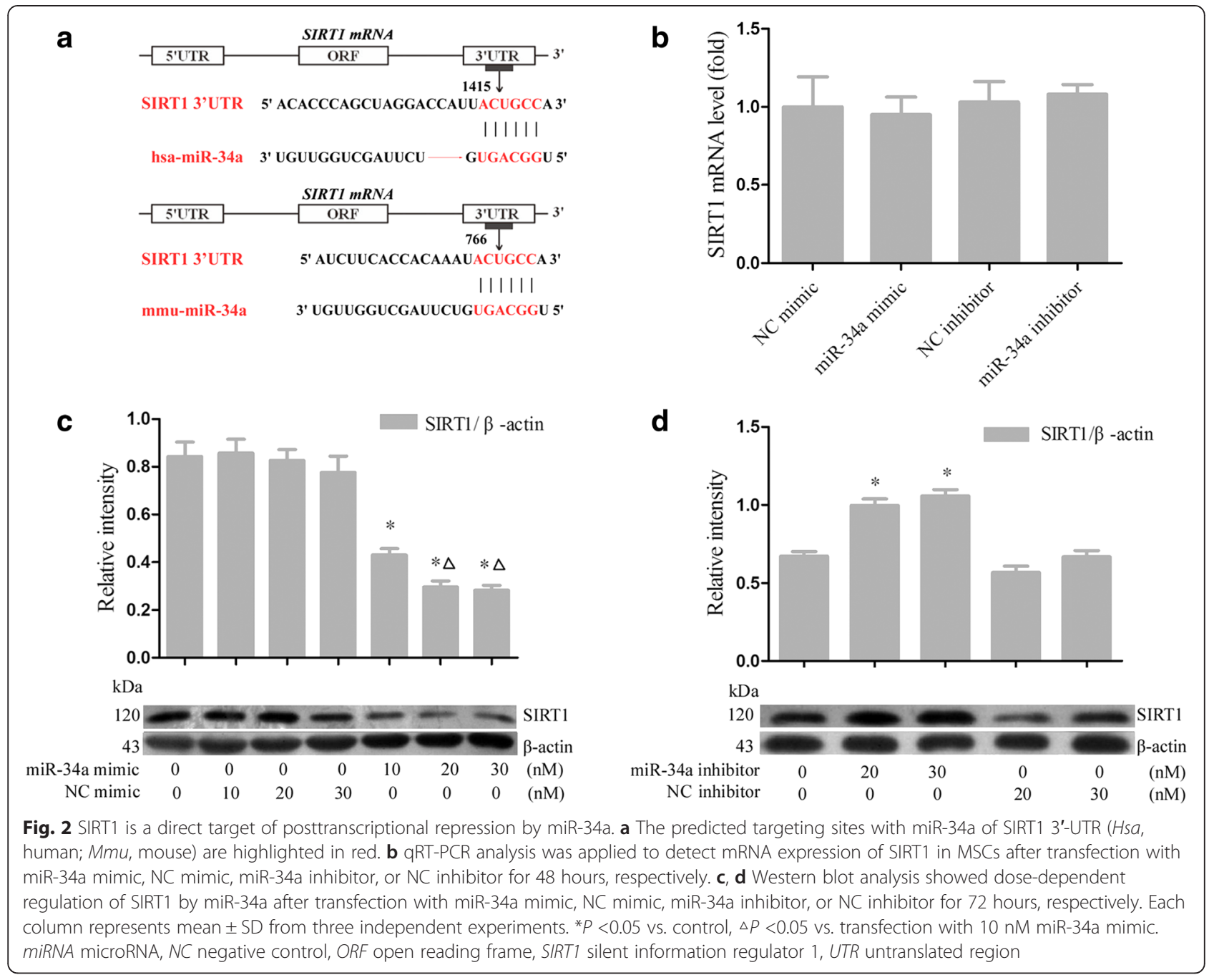

analysis of the percentage of cells that were Annexin $\mathrm{V}$ ${ }^{+} / \mathrm{PI}^{-}$(Fig. 3a, b). CASP3 is a well-studied mediator of apoptosis, because it is either partially or totally responsible for the cleavage of many key proteins, such as PARP1 [18]. In this study, increased activities of the cleaved CASP3 and cleaved PARP1 were observed when SIRT1 was knocked down or miR-34a was overexpressed (Fig. 3b, d). These findings suggest that knockdown of SIRT1 or treatment with miR-34a mimic acts similarly in the regulation of apoptosis.

To further elaborate the relationship between miR-34a and SIRT1, MSCs were transfected with miR-34a inhibitor or siRNA-SIRT1, or a combination, before exposure to H/SD. The results showed miR-34a inhibitor reduced CASP3 activity and expression of cleaved PARP1 (Fig. 3e, f), while siRNA-SIRT1 partly abolished the effects of the miR-34a inhibitor, verified both by flow cytometric analysis of the percentage of Annexin $\mathrm{V}^{+} / \mathrm{PI}^{-}$cells (Fig. 3a, b) and by western blot analysis of cleaved CASP3 and cleaved PARP1 (Fig. 3e, f).
SIRT1 plays important roles in many pathophysiological processes by deacetylating various substrates, including FOXO3, which has been reported to promote apoptosis by regulating its downstream target, the well-known proapoptotic protein Bim [24]. Our work revealed that downregulation of miR-34a decreased total FOXO3a and Bim protein expression, whereas SIRT1 knockdown increased the expression of these two proteins (Fig. 3e, f). However, neither miR34a inhibitor nor siRNA-SIRT1 altered the mRNA level of FOXO3a (data not shown), indicating that miR-34a and SIRT1 could regulate FOXO3a posttranscriptional activity under H/SD conditions. Our study revealed that the activation of SIRT1/FOXO3a and the intrinsic apoptosis pathway of CASP3-PARP1 might be involved in the pro-apoptotic function of miR-34a.

miR-34a exerts pro-apoptotic effects via activation of the mitochondrial apoptosis pathway

$\Delta \Psi \mathrm{m}$ is the main parameter of mitochondrial function used as an indicator of cell health [29]. Therefore, to 


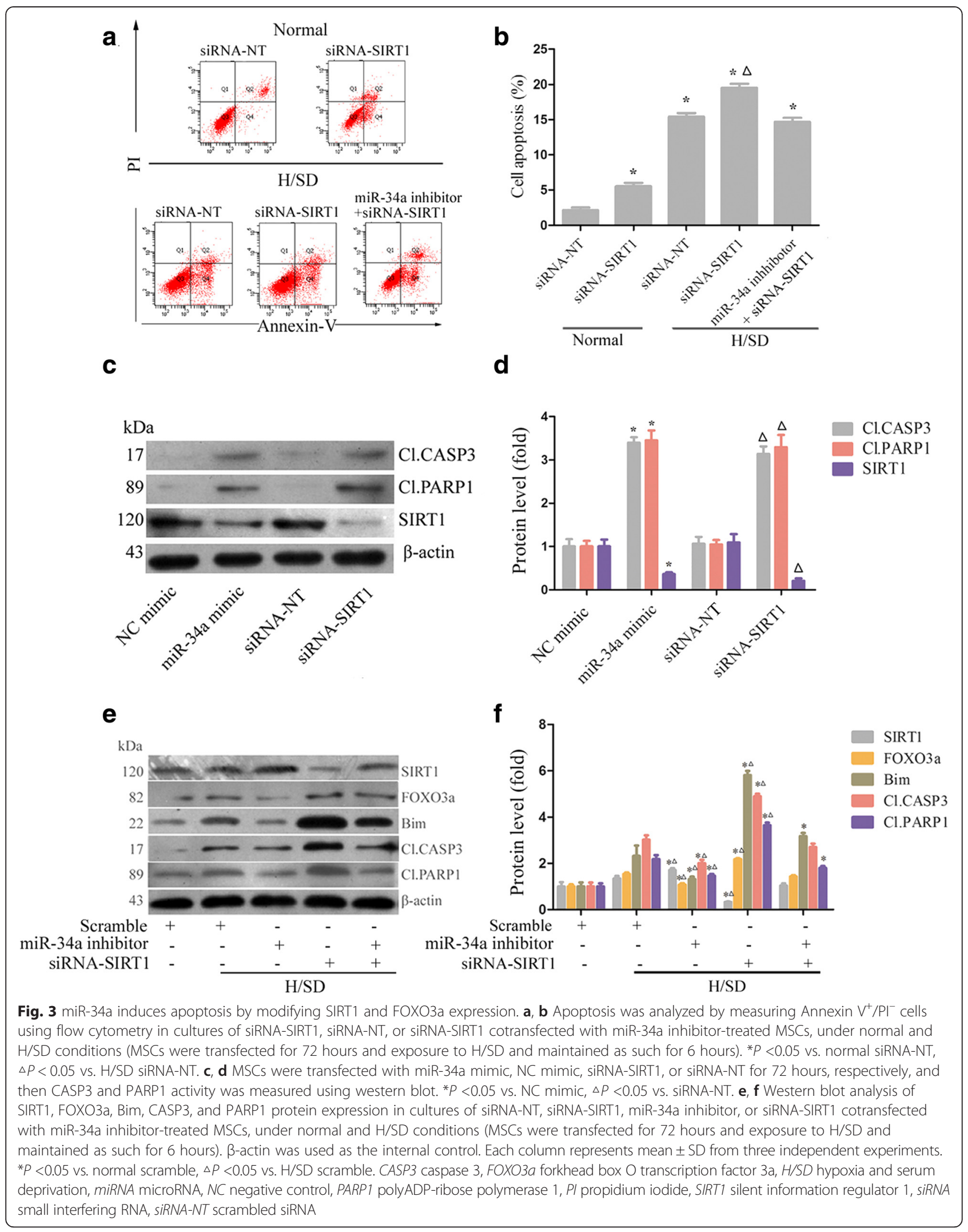


understand the intrinsic apoptotic pathway that is activated by miR-34a, we performed JC-1 staining. In contrast to the scramble cells, cells treated with the miR-34a inhibitor displayed significant changes in $\Delta \Psi \mathrm{m}$ (Fig. 4a). To further ascertain the effect of miR-34a, cellular fractionation was performed and cell lysates from cytosolic and mitochondrial fractions were subjected to western blotting to detect the expression of cytochrome $c$, which was released from mitochondria and functioned as a key mediator of apoptosis [30]. Western blot analysis revealed an inhibition of cytochrome $c$ release in the miR34a inhibitor group, while siRNA-SIRT1 reversed its effect (Fig. 4b, c). Taken together, these data support the hypothesis that miR-34a may be involved in the apoptotic process of MSCs induced by H/SD through activation of the mitochondrial apoptosis pathway by targeting SIRT1.

\section{Overexpression of miR-34a induces senescence in MSCs}

Considering that the regenerative capacity of MSCs contributed greatly to their function, we further examined cellular senescence in miR-34a mimic-transfected MSCs. SA- $\beta$-gal activity, which is a characteristic feature of senescence-related growth arrest [31], was assayed. Results revealed that overexpression of miR-34a significantly increased the percentage of SA- $\beta$-gal-positive cells compared with that of scramble (Fig. $5 \mathrm{a}, \mathrm{b}$ ). SIRT1 inhibition has been reported to be associated with premature senescence and impaired proliferative activity in EPCs [17]. Consistently, the percentage of SA- $\beta$-galpositive senescent cells was remarkably increased following SIRT1 knockdown (Fig. 5a, b).

ROS have been reported to induce oxidative stress that causes DNA and cell damage and can induce cell senescence through the p53/p21 pathway [32]. As expected, the miR-34a mimic increased ROS production, which was alleviated by addition of the ROS scavenger $N$-acetylcysteine (NAC) (Fig. 5c, d). $\gamma$-H2A.X, a sensitive marker for the formation of DNA damage foci, was examined by western blotting together with the senescence-related proteins p53, p21, and p16. In the miR-34a mimic and siRNASIRT1 group, the expression of p16, p53, and p21 was obviously increased compared with that in the control group (Fig. 5e, f). However, when ROS was removed by NAC or when miR-34a was inhibited by a miR-34a inhibitor, the expression of $\gamma$-H2A.X, p16, p53, and p21 was significantly reduced (Fig. 5e, f).

\section{Discussion}

Our results show that miR-34a is significantly upregulated under H/SD conditions in MSCs, and overexpression of miR-34a is strongly associated with increased apoptosis, lower viability, and increased senescence. SIRT1, identified as a direct and functional target of miR-34a, protects MSCs from H/SD-induced apoptosis through its downstream effector FOXO3a. Further

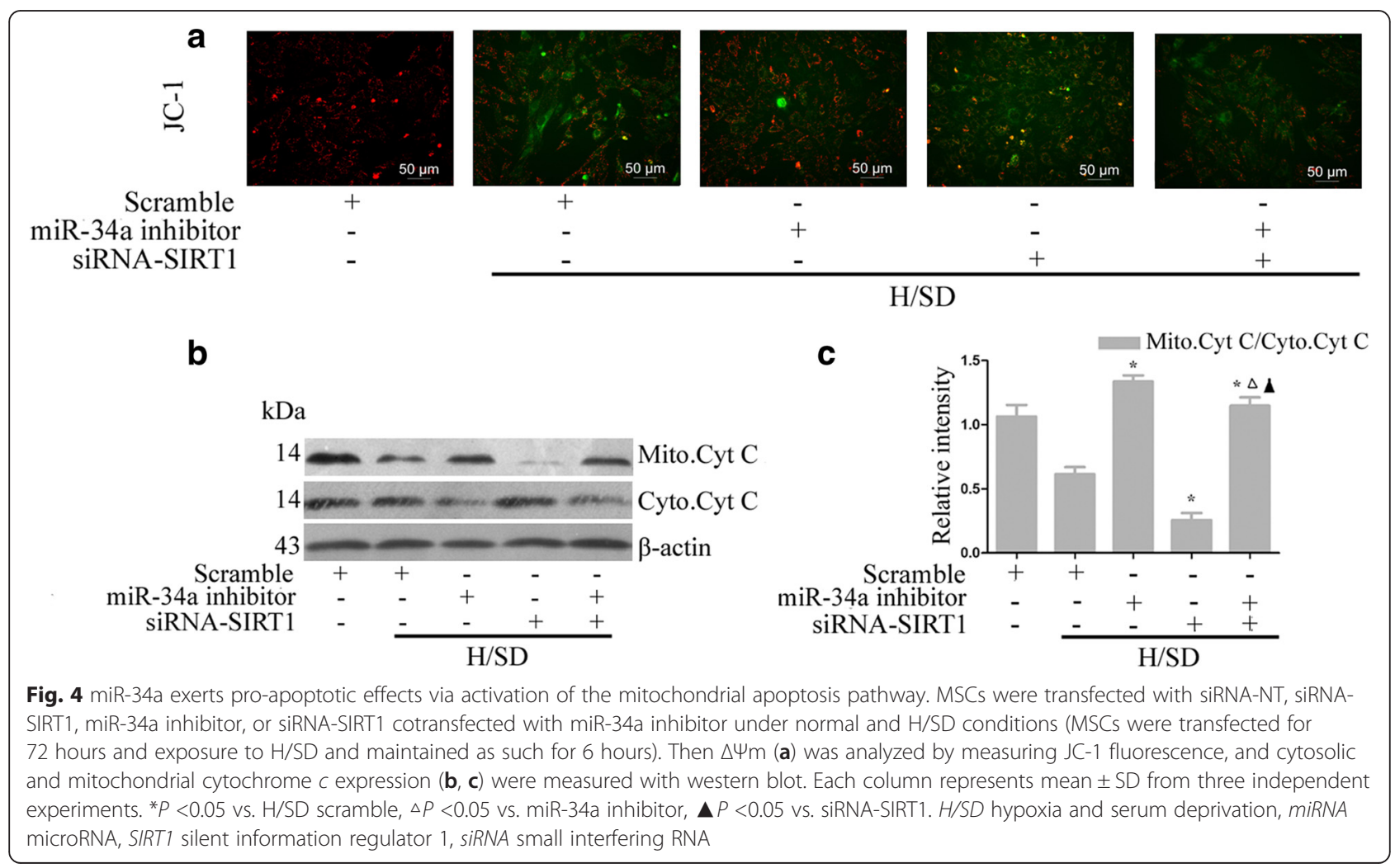


a

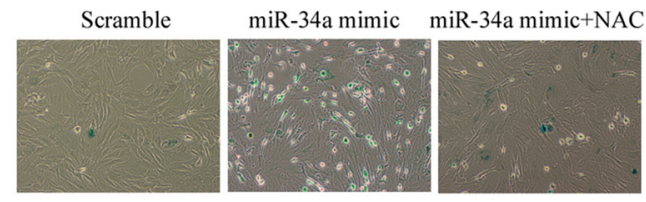

SiRNA-SIRT1 SiRNA-SIRT1+miR-34a inhibitor

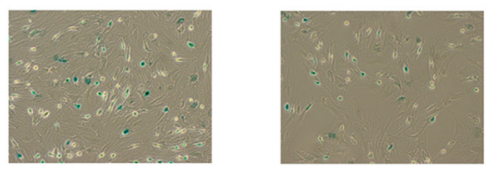

C

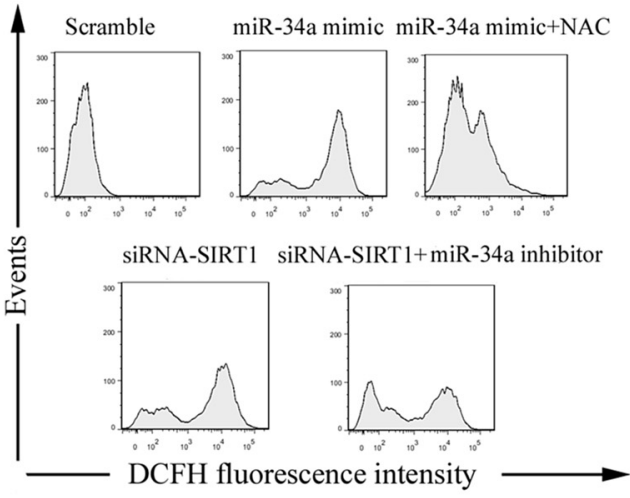

e

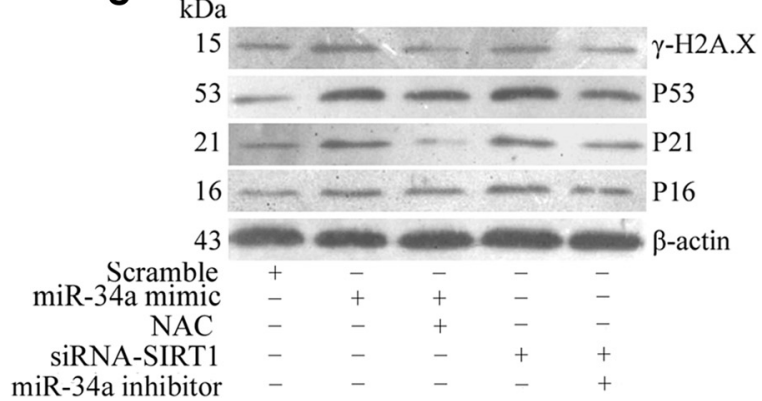

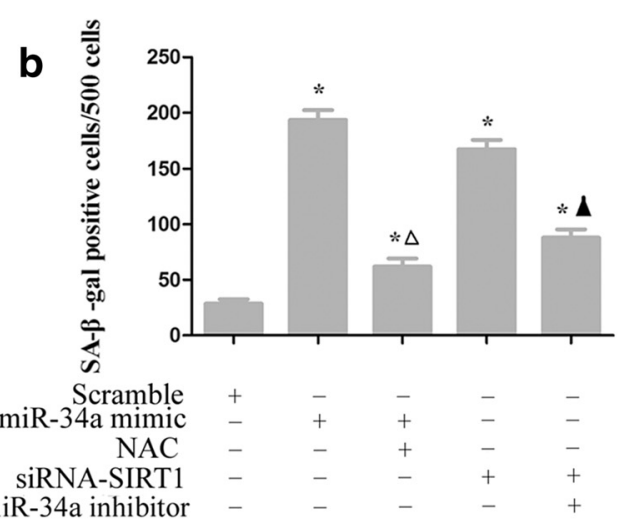

d

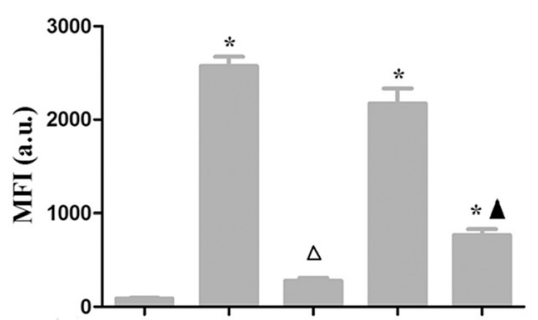

$\begin{array}{rlllll}\text { Scramble } & + & - & - & - & - \\ \text { miR-34a mimic } & - & + & + & - & - \\ \text { NAC } & - & - & + & - & - \\ \text { siRNA-SIRT1 } & - & - & - & + & + \\ \text { miR-34a inhibitor } & - & - & - & - & +\end{array}$

$\mathbf{f}$

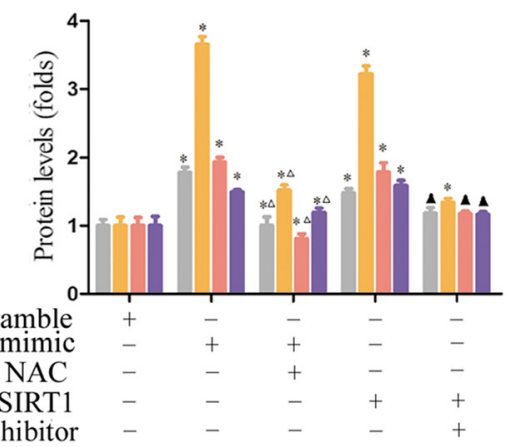

Fig. 5 Overexpression of miR-34a induces senescence in MSCs. Cells were left untreated or pretreated with miR-34a mimic, siRNA-SIRT1, ROS scavenger NAC (10 mM), and miR-34a inhibitor separately or in combination for 72 hours, and then cellular senescence was analyzed by SA- $\beta$-gal staining $(\mathbf{a}, \mathbf{b})$. Cellular ROS production was assessed by measuring the fluorescent intensity of DCFH-DA determined using flow cytometry (c, $\mathbf{d})$. Cellular DNA damage and senescence-related proteins including $\mathrm{Y}_{\mathrm{H}} \mathrm{H}_{2} \mathrm{~A} . \mathrm{X}, \mathrm{p} 53, \mathrm{p} 21$, and p16 were determined with western blot (e, f). Each column represents mean $\pm \mathrm{SD}$ from three independent experiments. ${ }^{*} P<0.05$ vs. scramble, $\triangle P<0.05$ vs. miR-34a mimic, $\Delta P<0.05$ vs. siRNA-SIRT1.cp. DCFH 2',7'-dichlorodihydrofluorescein, MFI mean fluorescence intensity, miRNA microRNA, NAC N-acetylcysteine, SA- $\beta$-gal senescence-associated galactosidase, SIRT1 silent information regulator 1, siRNA small interfering RNA

experiments indicated that the mitochondrial permeability transition and intrinsic apoptosis pathway of the CASP3-PARP1 axis were involved during this process. Moreover, miR-34a was also found to aggravate the senescence of MSCs in a ROS-dependent manner. This study strongly suggests that miR-34a is a promising candidate for a more optimized and appealing target for MSC-based therapy in MI.
Since MSCs are easily obtained and exhibit impressive paracrine ability as well as multilineage differentiation potential [33], autologous MSCs offer a great advantage when transplanted into ischemic or infarcted heart to regenerate and repopulate the injured myocardium and restore heart function. However, repair of cardiomyocytes and restoration of heart function are limited by poor survival [34], increased senescence [35], and loss of 
immunoprivilege in long-term preclinical studies of engrafted MSCs in the infarcted area [36]. Researchers have attempted numerous approaches to overcome these limitations, and have made some improvements in restoring cardiac function [37, 38]. Despite these successes, strategies are still needed to make transplanting MSCs into the infarcted area easier and more effective.

Recent observations have revealed that miRNAs are involved in the processes of apoptosis, proliferation, senescence, autophagy, and differentiation, among others, exhibiting powerful and unexpected roles in modulating cell biological functions by upregulating or downregulating these processes $[18,39]$. Among the known miRNAs, miR-34a has been demonstrated to be involved in apoptosis [40] and senescence [17] and to inhibit various key regulators of cell cycle progression [41]. miR-34a belongs to one of several evolutionarily-conserved families of miRNAs, namely miR-34, and was originally identified as a TP53-targeted mRNA [40]. miR-34a was expressed in almost every tissue but was scarcely expressed in lung tissue [42]. Frequently downregulated and functioning as an independent prognostic indicator in multiple types of cancers $[18,43]$, miR-34a expression levels were significantly upregulated in the animal model of acute MI and in the aged hearts [15] and were strongly correlated with left ventricular end-diastolic dimension 1 year after acute MI [44]. Furthermore, overexpression of miR-34a was demonstrated to promote the apoptosis of myocardial cell during MI [45], aggravate senescence, and impede angiogenesis ability of EPCs [17] and endothelial cells [46], as well as induce senescence and inflammation in vascular smooth muscle cells [47], leading to myocardial and vascular dysfunctions. Delivery of antagomir Ant-34a or LNA-based anti-miRNAs, however, enhanced cardiac contractile recovery after acute MI, which was associated with reduced fibrosis, increased capillary density, and elevated myocardial cell functions $[14,15]$. Thus, we presumed that miR-34a may play a crucial role in the MI microenvironment and contribute to the poor survival rate of MSCs in the infarcted area. In the present study, we showed that miR-34a was expressed in normal MSCs and was elevated greatly during $\mathrm{H} / \mathrm{SD}$, designed to mimic the in vivo conditions of ischemia and hypoxia. Overexpression of miR-34a aggravated MSC apoptosis, while inhibition of miR-34a expression conferred resistance to H/SD-induced apoptosis. These data suggests that miR-34a not only regulates resident myocardial cell apoptosis but also plays an important role in engrafted MSCs survival in the infarcted area.

SIRT1, one of the potential targets of miR-34a, has been identified as an apoptosis inhibitor and has been found to act as a longevity gene in many studies reported previously [48]. Recently, SIRT1 was reported to inhibit the apoptosis of vascular adventitial fibroblasts
(VAFs) [49]. Consistent with this report, our results showed that when SIRT1 was knocked down by siRNASIRT1, the apoptosis of MSCs induced by H/SD increased. However, we found a moderate increase in the expression of SIRT1 protein level in MSCs during H/SD (Fig. 3e), which was also found in EPCs exposed to $\mathrm{H}_{2} \mathrm{O}_{2}$ [24]. As is well known, SIRT1 is an NADdependent deacetylase, and the balance between NAD $+/ \mathrm{NADH}$ is crucial for cellular survival. A recent study demonstrated that during hypoxic and ischemic insults the concentration of $\mathrm{NAD}^{+}$increases at the very beginning, leading to activation of the SIRT1-dependent cleavage of acetyl groups [50]. This compensation effect can partly explain our result of the increased SIRT1 expression during $\mathrm{H} / \mathrm{SD}$.

In response to oxidative stress, SIRT1 forms a complex with FOXO3 and then enhances cellular stress resistance [51]. FOXO3a is a member of the mammalian FOXO family of forkhead transcription factors, which are critical regulators of stress responses, oncogenesis, and longevity by directly regulating the genes involved in apoptosis, cell cycle progression, and stress responses [52]. FOXO activities can be regulated by phosphorylation, ubiquitination, as well as deacetylation to inhibit apoptosis $[25,51,53]$. It has been identified that SIRT1 can bind to and deacetylate FOXO3a and then suppress its transcriptional activity, which also exerts favorable effects on oxidative stress resistance in cardiac myocytes [51]. In the current study, we showed in MSCs that FOXO3a was downregulated in response to a miR-34a inhibitor, and could be abolished by silencing SIRT1 expression, suggesting that the restorative function of the miR-34a inhibitor in MSCs was mediated through the SIRT1-FOXO3a signaling pathway. Regulation of the SIRT1-FOXO3a signaling pathway through some basic strategies may therefore play an active role in clinical heart disease.

We further examined the role of $\triangle \Psi \mathrm{m}$ and the intrinsic apoptosis pathway of the CASP3-PARP1 axis in the pro-apoptotic activity of miR-34a in MSCs. Considerable evidence implicated that mitochondrial dysfunction or a change in the $\Delta \Psi \mathrm{m}$ was one of the signs of cell death [28]. The decrease of $\Delta \Psi \mathrm{m}$ activates effector CASP3 by a series of reactions, and subsequently induces cell apoptosis. As expected, we found that inhibition of miR-34a increased the $\Delta \Psi \mathrm{m}$ of MSCs during $\mathrm{H} / \mathrm{SD}$, and decreased cleaved CASP3 expression.

Like other cells, MSCs entered into the senescence process when exposed to oxidative stress which greatly reduced their regenerative capacity and limited their transplantation efficiency. As an inevitable by-product of mitochondrial respiration, ROS in moderate amounts is necessary for cell survival, proliferation, and longevity [54]. However, during hypoxia, an imbalance between 
the formation and scavenging of free radicals leads to overproduction of electrons. These electrons react with remnant molecular oxygen, leading to ROS generation [55]. Abundant ROS could result in cell senescence through inducing DNA damage [54]. In this study, we explored whether ROS was the main mediator of MSC senescence induced by miR-34a overexpression. Results showed that overexpression of miR-34a increased $\beta$ galactosidase-positive cells as well as ROS production. When ROS production was reduced by NAC in MSCs, DNA damage was attenuated and the expression of p16, p53, and p21 was reduced. These results imply that ROS has an important role in MSC senescence induced by miR-34a overexpression and thus may partly explain the poor survival rate of engrafted MSCs in the infarcted area.

With rapid development of anti-miRNA chemistries, even ahead of miRNA mimicry [56], the miR-34a knockdown by antagomirs or LNA-based anti-miRNAs has been shown to protect against the deterioration of cardiac systolic function in mice after acute MI [15]. Moreover, no side effect was reported. Intracoronary infusion or intramyocardial delivery of MSCs modified with current developing therapeutics to inhibit the expression of miR-34a might thus have great advantage in application for vascular diseases.

In conclusion, our study reveals that miR-34a is greatly elevated in MSCs during H/SD, and overexpression of miR-34a leads to robust apoptosis, while inhibition of miR-34a significantly increases pressure resistance of MSCs to H/SD. This apoptosis is highly regulated by SIRT1/FOXO3a pathway activation, mitochondrial dysfunction, and finally activation of the intrinsic apoptosis pathway of the CASP3-PARP1 axis. Moreover, our results show that miR-34a overexpression increases cellular senescence, which may be regulated by ROS production. However, more work will be needed to determine the role of SIRT1 and FOXO3a in miR-34a-mediated functions in myocardial ischemia in vivo and to investigate whether other signaling pathways such as $\mathrm{NOTCH}$ signaling pathways, which have been reported to be regulated by miR-34a [57], are involved in the H/SD process in MSCs.

\section{Conclusions}

Our data demonstrate that miR-34a is involved in the process of H/SD in MSCs, while inhibition of miR-34a leads to an increase in SIRT1 and a decrease in FOXO3a protein expression, fewer apoptotic cells, and better viability. Moreover, we found that overexpression of miR34a induced senescence of MSCs, which may partly be abolished by the ROS scavenger NAC. Inhibition of miR-34a in MSCs would thus be beneficial and could demonstrate great therapeutic potential in clinical transplantation for vascular disorders.

\section{Abbreviations}

$\triangle \Psi \mathrm{m}$ : Mitochondrial membrane potential; BCA: Bicinchoninic acid assay; CASP3: Caspase 3; CCK-8: Cell counting kit-8; DCFH-DA:

2',7'-Dichlorodihydrofluorescein diacetate; EPC: Endothelial progenitor cell; FBS: Fetal bovine serum; FITC: Fluorescein isothiocyanate; FOXO3a: Forkhead box O transcription factor 3a; GAPDH: Glyceraldehyde 3-phosphate dehydrogenase; H/SD: Hypoxia and serum deprivation; IHD: Ischemic heart disease; IMDM: Iscove's modified Dulbecco's medium; MI: Myocardial infarction; miRNA: MicroRNA; MSC: Mesenchymal stem cell; NAC: Nacetylcysteine; NC: Negative control; PARP1: polyADP-ribose polymerase 1; PBS: Phosphate-buffered saline; PI: Propidium iodide; PVDF: Polyvinylidene difluoride; qRT-PCR: Quantitative RT-PCR; ROS: Reactive oxygen species; SA- $\beta$-gal: Senescence-associated $\beta$-galactosidase; SD: Standard deviation; SDS: Sodium dodecyl sulfate; siRNA: Small interfering RNA; siRNANT: Scrambled siRNA; SIRT1: Silent information regulator 1; TBS: Tris-buffered saline; TBS-T: TBS with Tween-20; UTR: Untranslated region; VAF: Vascular adventitial fibroblast.

\section{Competing interests}

The authors declare that there are no competing interests.

\section{Authors' contributions}

FZ contributed to the experimental design, carried out the molecular biology experiments, performed the statistical analysis, and drafted the manuscript. JC participated in the design of the study and the sequence alignment and revised the manuscript. XiaL was responsible for MSC transfection, statistical analysis, and helped to draft the manuscript. BL carried out the immunoassays and revised the manuscript. XinL participated in isolation and culture of MSCs and helped to draft the manuscript. ZX participated in the design of the study, performed the statistical analysis, and helped to revise the manuscript. BY conceived of the study, and participated in its design and coordination and helped to draft the manuscript. All authors read and approved the final manuscript.

\section{Acknowledgements}

This study was supported by grants from the National Natural Science Foundation of China (to BY, grant numbers 81171430 and 81330033; to BL, grant number 81400296 ) and the Key Laboratory of Myocardial Ischemia Mechanism and Treatment (Harbin Medical University), Ministry of Education (to XiaL, grant number KF201412).

\section{Author details}

${ }^{1}$ Key Laboratories of Education Ministry for Myocardial Ischemia Mechanism, The Second Affiliated Hospital of Harbin Medical University, 148 Baojian Road, Harbin 150086, P.R. China. ${ }^{2}$ Department of Cardiology, The Second Affiliated Hospital of Harbin Medical University, 148 Baojian Road, Harbin 150086, P.R. China. ${ }^{3}$ Department of Cardiology, Mudanjiang Forestry Central Hospital, 50 XinhuaRoad, Mudanjiang 157000, P.R. China.

Received: 23 July 2015 Revised: 13 August 2015

Accepted: 21 September 2015 Published online: 07 October 2015

\section{References}

1. Moran AE, Forouzanfar MH, Roth GA, Mensah GA, Ezzati M, Murray CJ, et al. Temporal trends in ischemic heart disease mortality in 21 world regions, 1980 to 2010: the Global Burden of Disease 2010 study. Circulation. 2014;129:1483-92.

2. McMurray JJ. Clinical practice. Systolic heart failure. N Engl J Med. 2010;362:228-38.

3. Quevedo HC, Hatzistergos KE, Oskouei BN, Feigenbaum GS, Rodriguez JE, Valdes $D$, et al. Allogeneic mesenchymal stem cells restore cardiac function in chronic ischemic cardiomyopathy via trilineage differentiating capacity. Proc Natl Acad Sci U S A. 2009;106:14022-7.

4. Karantalis V, DiFede DL, Gerstenblith G, Pham S, Symes J, Zambrano JP, et al. Autologous mesenchymal stem cells produce concordant improvements in regional function, tissue perfusion, and fibrotic burden when administered to patients undergoing coronary artery bypass grafting: the Prospective Randomized Study of Mesenchymal Stem Cell Therapy in Patients Undergoing Cardiac Surgery (PROMETHEUS) trial. Circ Res. 2014;114:1302-10. 
5. Leistner DM, Fischer-Rasokat U, Honold J, Seeger FH, Schachinger V, Lehmann $\mathrm{R}$, et al. Transplantation of progenitor cells and regeneration enhancement in acute myocardial infarction (TOPCARE-AMI): final 5-year results suggest long-term safety and efficacy. Clin Res Cardiol. 2011;100:925-34.

6. Toma C, Pittenger MF, Cahill KS, Byrne BJ, Kessler PD. Human mesenchymal stem cells differentiate to a cardiomyocyte phenotype in the adult murine heart. Circulation. 2002;105:93-8.

7. Pagani FD, DerSimonian H, Zawadzka A, Wetzel K, Edge AS, Jacoby DB, et al. Autologous skeletal myoblasts transplanted to ischemia-damaged myocardium in humans. Histological analysis of cell survival and differentiation. J Am Coll Cardiol. 2003:41:879-88.

8. Burova E, Borodkina A, Shatrova A, Nikolsky N. Sublethal oxidative stress induces the premature senescence of human mesenchymal stem cells derived from endometrium. Oxidative Med Cell Longev. 2013;2013:474931.

9. van Rooij E, Purcell AL, Levin AA. Developing microRNA therapeutics. Circ Res. 2012;110:496-507.

10. Bernardo BC, Charchar FJ, Lin RC, McMullen JR. A microRNA guide for clinicians and basic scientists: background and experimental techniques. Heart Lung Circ. 2012;21:131-42.

11. Small EM, Olson EN. Pervasive roles of microRNAs in cardiovascular biology. Nature. 2011;469:336-42.

12. Lin RC, Weeks KL, Gao XM, Williams RB, Bernardo BC, Kiriazis H, et al. PI3K(p110 alpha) protects against myocardial infarction-induced heart failure: identification of PI3K-regulated miRNA and mRNA. Arterioscler Thromb Vasc Biol. 2010;30:724-32.

13. Greco S, Fasanaro P, Castelvecchio S, D'Alessandra Y, Arcelli D, Di Donato M, et al. MicroRNA dysregulation in diabetic ischemic heart failure patients. Diabetes. 2012;61:1633-41.

14. Bernardo BC, Gao XM, Winbanks CE, Boey EJ, Tham YK, Kiriazis H, et al. Therapeutic inhibition of the miR-34 family attenuates pathological cardiac remodeling and improves heart function. Proc Natl Acad Sci U S A. 2012;109:17615-20.

15. Boon RA, lekushi K, Lechner S, Seeger T, Fischer A, Heydt S, et al. MicroRNA34a regulates cardiac ageing and function. Nature. 2013:495:107-10.

16. Krzeszinski JY, Wei W, Huynh H, Jin Z, Wang X, Chang TC, et al. miR-34a blocks osteoporosis and bone metastasis by inhibiting osteoclastogenesis and Tgif2. Nature. 2014;512:431-5.

17. Zhao T, Li J, Chen AF. MicroRNA-34a induces endothelial progenitor cell senescence and impedes its angiogenesis via suppressing silent information regulator 1. Am J Physiol Endocrinol Metab. 2010;299:E110-6.

18. Liu K, Huang J, Xie M, Yu Y, Zhu S, Kang R, et al. MIR34A regulates autophagy and apoptosis by targeting HMGB1 in the retinoblastoma cell. Autophagy. 2014;10:442-52.

19. Yamakuchi M, Ferlito M, Lowenstein CJ. miR-34a repression of SIRT1 regulates apoptosis. Proc Natl Acad Sci U S A. 2008;105:13421-6.

20. Lai L, Yan L, Gao S, Hu CL, Ge H, Davidow A, et al. Type 5 adenylyl cyclase increases oxidative stress by transcriptional regulation of manganese superoxide dismutase via the SIRT1/FoxO3a pathway. Circulation. 2013;127:1692-701.

21. Mouchiroud L, Houtkooper RH, Moullan N, Katsyuba E, Ryu D, Canto C, et al. The $\mathrm{NAD}(+)$ /sirtuin pathway modulates longevity through activation of mitochondrial UPR and FOXO signaling. Cell. 2013;154:430-41.

22. Li M, Chiu JF, Mossman BT, Fukagawa NK. Down-regulation of manganesesuperoxide dismutase through phosphorylation of FOXO3a by Akt in explanted vascular smooth muscle cells from old rats. J Biol Chem. 2006:281:40429-39.

23. Warr MR, Binnewies M, Flach J, Reynaud D, Garg T, Malhotra R, et al. FOXO3A directs a protective autophagy program in haematopoietic stem cells. Nature. 2013;494:323-7.

24. Wang $Y Q$, Cao Q, Wang F, Huang LY, Sang TT, Liu F, et al. SIRT1 protects against oxidative stress-induced endothelial progenitor cells apoptosis by inhibiting FOXO3a via FOXO3a ubiquitination and degradation. J Cell Physiol. 2015;230:2098-107

25. Xia W, Zhang F, Xie C, Jiang M, Hou M. Macrophage migration inhibitory factor confers resistance to senescence through CD74-dependent AMPKFOXO3a signaling in mesenchymal stem cells. Stem Cell Res Ther. 2015;6:82.

26. Zhu W, Chen J, Cong X, Hu S, Chen X. Hypoxia and serum deprivationinduced apoptosis in mesenchymal stem cells. Stem Cells. 2006:24:416-25.

27. Wang XQ, Shao Y, Ma CY, Chen W, Sun L, Liu W, et al. Decreased SIRT3 in aged human mesenchymal stromal/stem cells increases cellular susceptibility to oxidative stress. J Cell Mol Med. 2014;18:2298-310.
28. Deuse T, Peter C, Fedak PW, Doyle T, Reichenspurner H, Zimmermann WH, et al. Hepatocyte growth factor or vascular endothelial growth factor gene transfer maximizes mesenchymal stem cell-based myocardial salvage after acute myocardial infarction. Circulation. 2009;120:S247-54.

29. Duchen MR. Roles of mitochondria in health and disease. Diabetes. 2004:53:S96-102.

30. Garrido C, Galluzzi L, Brunet M, Puig PE, Didelot C, Kroemer G. Mechanisms of cytochrome c release from mitochondria. Cell Death Differ. 2006;13:1423-33.

31. Dimri GP, Lee X, Basile G, Acosta M, Scott G, Roskelley C, et al. A biomarker that identifies senescent human cells in culture and in aging skin in vivo. Proc Natl Acad Sci U S A. 1995;92:9363-7.

32. Wang H, Zhou W, Zheng Z, Zhang P, Tu B, He Q, et al. The HDAC inhibitor depsipeptide transactivates the p53/p21 pathway by inducing DNA damage. DNA Repair. 2012;11:146-56

33. Frenette PS, Pinho S, Lucas D, Scheiermann C. Mesenchymal stem cell: keystone of the hematopoietic stem cell niche and a stepping-stone for regenerative medicine. Annu Rev Immunol. 2013;31:285-316.

34. Clifford DM, Fisher SA, Brunskill SJ, Doree C, Mathur A, Watt S, et al. Stem cell treatment for acute myocardial infarction. Cochr Database Syst Rev. 2012;2:CD006536.

35. Ni NC, Li RK, Weisel RD. The promise and challenges of cardiac stem cell therapy. Semin Thorac Cardiovasc Surg. 2014;26:44-52.

36. Huang XP, Sun Z, Miyagi Y, McDonald Kinkaid H, Zhang L, Weisel RD, et al. Differentiation of allogeneic mesenchymal stem cells induces immunogenicity and limits their long-term benefits for myocardial repair. Circulation. 2010;122:2419-29.

37. Shahzad U, Li G, Zhang Y, Li RK, Rao V, Yau TM. Transmyocardial revascularization enhances bone marrow stem cell engraftment in infarcted hearts through SCF-Ckit and SDF-1-CXCR4 signaling axes. Stem Cell Rev. 2015;11:332-46.

38. Dhingra S, Li P, Huang XP, Guo J, Wu J, Mihic A, et al. Preserving prostaglandin E2 level prevents rejection of implanted allogeneic mesenchymal stem cells and restores postinfarction ventricular function. Circulation. 2013;128:569-78.

39. Jheon AH, Li CY, Wen T, Michon F, Klein OD. Expression of microRNAs in the stem cell niche of the adult mouse incisor. PLoS One. 2011;6, e24536.

40. He L, He X, Lim LP, de Stanchina E, Xuan Z, Liang Y, et al. A microRNA component of the p53 tumour suppressor network. Nature. 2007;447:1130-4.

41. Tarasov $V$, Jung $P$, Verdoodt $B$, Lodygin D, Epanchintsev A, Menssen A, et al. Differential regulation of microRNAs by $\mathrm{p} 53$ revealed by massively parallel sequencing: miR-34a is a p53 target that induces apoptosis and G1-arrest. Cell Cycle. 2007;6:1586-93.

42. Bommer GT, Gerin I, Feng Y, Kaczorowski AJ, Kuick R, Love RE, et al. p53-mediated activation of miRNA34 candidate tumor-suppressor genes. Curr Biol. 2007:17:1298-307.

43. Asslaber D, Pinon JD, Seyfried I, Desch P, Stocher M, Tinhofer I, et al. microRNA-34a expression correlates with MDM2 SNP309 polymorphism and treatment-free survival in chronic lymphocytic leukemia. Blood. 2010;115:4191-7.

44. Matsumoto S, Sakata Y, Suna S, Nakatani D, Usami M, Hara M, et al. Circulating p53-responsive microRNAs are predictive indicators of heart failure after acute myocardial infarction. Circ Res. 2013;113:322-6.

45. Fan F, Sun A, Zhao H, Liu X, Zhang W, Jin X, et al. MicroRNA-34a promotes cardiomyocyte apoptosis post myocardial infarction through down-regulating aldehyde dehydrogenase 2. Curr Pharm Des. 2013;19:4865-73.

46. Ito T, Yagi S, Yamakuchi M. MicroRNA-34a regulation of endothelial senescence. Biochem Biophys Res Commun. 2010;398:735-40.

47. Badi I, Burba I, Ruggeri C, Zeni F, Bertolotti M, Scopece A et al. MicroRNA-34a induces vascular smooth muscle cells senescence by SIRT1 downregulation and promotes the expression of age-associated pro-inflammatory secretory factors. J Gerontol Ser A Biol Sci Med Sci. 2014. doi: 10.1093/gerona/glu180. Epub ahead of print.

48. Gomes AP, Price NL, Ling AJ, Moslehi JJ, Montgomery MK, Rajman L, et al. Declining $N A D(+)$ induces a pseudohypoxic state disrupting nuclearmitochondrial communication during aging. Cell. 2013;155:1624-38.

49. Wang W, Yan C, Zhang J, Lin R, Lin Q, Yang L, et al. SIRT1 inhibits TNF-alpha-induced apoptosis of vascular adventitial fibroblasts partly through the deacetylation of FoxO1. Apoptosis. 2013;18:689-701.

50. Blander G, Guarente L. The Sir2 family of protein deacetylases. Annu Rev Biochem. 2004;73:417-35.

51. Brunet A, Sweeney LB, Sturgill JF, Chua KF, Greer PL, Lin Y, et al. Stress-dependent regulation of FOXO transcription factors by the SIRT1 deacetylase. Science. 2004;303:2011-5. 
52. Nakae J, Oki M, Cao Y. The FoxO transcription factors and metabolic regulation. FEBS Lett. 2008;582:54-67.

53. Wang $X$, Chen WR, Xing D. A pathway from JNK through decreased ERK and Akt activities for FOXO3a nuclear translocation in response to UV irradiation. J Cell Physiol. 2012;227:1168-78.

54. Yee C, Yang W, Hekimi S. The intrinsic apoptosis pathway mediates the pro-longevity response to mitochondrial ROS in C. elegans. Cell. 2014;157:897-909.

55. Acin-Perez R, Carrascoso I, Baixauli F, Roche-Molina M, Latorre-Pellicer A, Fernandez-Silva P, et al. ROS-triggered phosphorylation of complex II by Fgr kinase regulates cellular adaptation to fuel use. Cell Metab. 2014;19:1020-33.

56. van Rooij E, Olson EN. MicroRNA therapeutics for cardiovascular disease: opportunities and obstacles. Nat Rev Drug Discov. 2012;11:860-72.

57. Bu P, Chen KY, Chen JH, Wang L, Walters J, Shin YJ, et al. A microRNA miR-34a-regulated bimodal switch targets Notch in colon cancer stem cells. Cell Stem Cell. 2013;12:602-15.

\section{Submit your next manuscript to BioMed Central and take full advantage of:}

- Convenient online submission

- Thorough peer review

- No space constraints or color figure charges

- Immediate publication on acceptance

- Inclusion in PubMed, CAS, Scopus and Google Scholar

- Research which is freely available for redistribution 